\title{
DETERMINAÇÃO DA INSTABILIDADE CROMOSSÔMICA E MORTE CELULAR EM CÉLULAS DA MUCOSA BUCAL EM PACIENTES COM DEFICIÊNCIA COGNITIVA
}

\section{DETERMINATION OF CHROMOSOMIC INSTABILITY AND CELL DEATH IN ORAL MUCOSA CELLS IN PATIENTS WITH COGNITIVE DISABILITY}

Isabela Soares Uchôa ${ }^{1}$, Francisco Ariel Paz Santos Freitas ${ }^{2}$, Maria do Amparo Veloso Magalhães ${ }^{3}$, Suzanne Tatianne Teixeira Nascimento ${ }^{4}$, Matheus Fernandes de Castro ${ }^{5}$, Nelson Jorge Carvalho Batista ${ }^{6}$

Submetido em: 21/04/2021

e24217

Aprovado em: 01/05/2021

\section{RESUMO}

A aplicação do teste de micronúcleos ( $\mathrm{MN})$, utilizando células esfoliadas da cavidade bucal é amplamente usada no biomonitoramento de lesões orais pré-cancerígenas, levando em consideração que a cavidade oral pode refletir a saúde dos indivíduos. A presente pesquisa buscou determinar a instabilidade cromossômica e morte celular em células da mucosa bucal em pacientes com deficiência cognitiva atendidos em serviço de referência odontológica a pessoa com deficiência intelectual e autismo em Teresina-PI. Trata-se de um estudo qualitativo e quantitativo amostral que visa à coleta, e a análise de dados de material biológico. Fizeram parte da amostra 30 indivíduos sem restrição de gênero, com registro de atendimento de maio de 2017 a janeiro de 2019, que retornaram para reavaliação oral de rotina. Um grupo controle pareado de pacientes normosistêmicos também foi abordado $(n=30)$, após a assinatura do Termo de Consentimento Livre e Esclarecido (TCLE). Os resultados evidenciaram que a frequência de MN, bem como de outras alterações celulares foram maiores em pacientes com deficiência cognitiva e que esses achados podem se relacionar com as doenças de base. Conclui-se que a aplicação do teste de MN pode ser fundamental nesses pacientes para detectar os danos no DNA cromossômico e predizer os riscos para o câncer de boca, mas é imprescindível que haja a ampliação de estudos nessa área como forma de trazer melhorias na qualidade de vida dos mesmos, de modo a contribuir com aprimoramento das medidas de prevenção.

PALAVRAS-CHAVE: Instabilidade genômica. Micronúcleo. Doenças crônicas.

\footnotetext{
1 Discente do $8^{\circ}$ período do curso Bacharelado em Enfermagem no Centro Universitário Santo Agostinho UNIFSA. Atuou como monitora nas disciplinas de Anatomia Humana I e II, e em Semiologia e Semiotécnica. Atualmente é aluna bolsista do PIBIC com a realização de pesquisa na área de determinação de instabilidade cromossômica através da aplicação do teste de micronúcleos.

2 Graduando do 9 período do curso de Bacharelado em Enfermagem pelo Centro Universitário Santo Agostinho - UNIFSA; Técnico de Administração pelo Instituto Federal de Ciência e Tecnologia do Maranhão IFMA, Timon (MA).

3 Possui graduação em Odontologia pela Universidade Federal do Piauí (1992), graduação em Química pela Universidade Federal do Piauí (1985) e doutorado em Biologia Celular e Molecular Aplicada à Saúde pela Universidade Luterana do Brasil (2016). Atualmente é policial militar: oficial da saúde- cirurgiã do Centro Universitário Santo Agostinho de Teresina, estatutária - Polícia Militar do Piauí, colaborador da Associação Brasileira de Cirurgiões Dentistas - Secção Piauí e tempo parcial do Centro Universitário Santo Agostinho.

${ }^{4}$ Possui graduação em Serviço Social pela Faculdade Santo Agostinho de Teresina (2014). Tem experiência na área de Serviço Social.

5 Graduação em andamento em Enfermagem - Centro Universitário Santo Agostinho - UNIFSA

6 Graduado em Ciências Biológicas pela Universidade Estadual do Piauí, Especialista em Educação Ambiental pela Faculdade de Educação Superior do Piauí, Mestre em Genética e Toxicologia Aplicada pela Universidade Luterana do Brasil - ULBRA / RS, Doutor em Biologia Celular e Molecular Aplicada à Saúde Pela ULBRA / RS. Professor da Faculdade Santo Agostinho. Membro da equipe editorial da Revista Saúde em foco da Faculdade Santo Agostinho e da colombiana Acta Biológica.
} 


\section{RECIMA21 - REVISTA CIENTÍFICA MULTIDISCIPLINAR ISSN 2675-6218}

DETERMINAÇÃO DA INSTABILIDADE CROMOSSÔMICA E MORTE CELULAR EM CÉLULAS DA MUCOSA BUCAL EM PACIENTES COM DEFICIÊNCIA COGNITIVA Isabela Soares Uchôa, Francisco Ariel Paz Santos Freitas, Maria do Amparo Veloso Magalhães, Suzanne Tatianne Teixeira Nascimento, Matheus Fernandes de Castro, Nelson Jorge Carvalho Batista

\section{ABSTRACT}

The application of the micronucleus (MN) test, using exfoliated cells from the oral cavity, is widely used in the biomonitoring of precancerous oral lesions, taking into account that the oral cavity may reflect the health of individuals. This research sought to determine chromosomal instability and cell death in oral mucosa cells in patients with cognitive impairment treated at a dental reference service for people with intellectual disabilities and autism in Teresina-PI. This is a qualitative and quantitative sample study that aims to collect and analyze data on biological material. The sample included 30 individuals without gender restriction, with attendance records from May 2017 to January 2019, who returned for routine oral reevaluation. A matched control group of normosystemic patients was also approached $(n=30)$, after signing the Informed Consent Form (ICF). The results showed that the frequency of $M N$, as well as other cellular changes, were higher in patients with cognitive impairment and that these findings may be related to underlying diseases. It is concluded that the application of the MN test can be fundamental in these patients to detect the damage in chromosomal DNA and predict the risks for oral cancer, but it is essential that there is an expansion of studies in this area as a way to bring improvements in quality their lives, in order to contribute to the improvement of prevention measures.

KEYWORDS: Genomic instability. Micronucleus. Chronic diseases.

\section{INTRODUÇÃo}

A cognição é o ato ou processo da aquisição do conhecimento que se dá através da percepção, da atenção, associação, memória, raciocínio, juízo, imaginação, pensamento e linguagem (FERREIRA, 2010). É o conjunto dos processos mentais usados no pensamento na classificação, reconhecimento e compreensão para o julgamento através do raciocínio para 0 aprendizado de determinados sistemas e soluções de problemas. Alguns fatores como socioeconômico, psicológico e de saúde, podem contribuir para o declínio gradual das funções cognitivas (GURIAN et al., 2012).

Segundo os autores supracitados, as modificações e os déficits causados pela perda na cognição acarretam consequências diretas sobre a qualidade de vida das pessoas, podendo levar ao declínio funcional, com diminuição e/ou perdas das habilidades para o desenvolvimento das atividades da vida diárias (AVD). Sabe-se que tanto as doenças físicas como as mentais podem levar à perda da independência e autonomia, sendo fatores de risco importantes para a mortalidade

É nesse contexto que podemos ressaltar a importância do cuidador, que pode ser algum familiar ou profissional que desempenha um papel essencial na vida diária dos pacientes com deficiência cognitiva, envolvendo-se em praticamente todos os aspectos do cuidado e assumindo responsabilidades adicionais de maneira crescente (CRUZ; HAMDAN, 2008).

Vale ressaltar que as pessoas que têm como responsabilidade cuidar de pacientes especiais acabam tendo um impacto significativo em suas vidas. Ainda segundo Cruz e Hamdan. (2008), alguns resultados têm evidenciado que o declínio do paciente e suas demandas específicas são fatores importantes de estresse para o cuidador. Estes apresentam altos índices 


\section{RECIMA21 - REVISTA CIENTÍFICA MULTIDISCIPLINAR ISSN 2675-6218}

DETERMINAÇÃO DA INSTABILIDADE CROMOSSÔMICA E MORTE CELULAR EM CÉLULAS DA MUCOSA BUCAL EM PACIENTES COM DEFICIÊNCIA COGNITIVA Isabela Soares Uchôa, Francisco Ariel Paz Santos Freitas, Maria do Amparo Veloso Magalhães, Suzanne Tatianne Teixeira Nascimento, Matheus Fernandes de Castro, Nelson Jorge Carvalho Batista

de sintomas psiquiátricos - especialmente a depressão -, prejuízos no sistema imunológico, assim como altos índices de conflitos familiares. Outros estudos sugerem que altos índices de sobrecarga e impacto no cuidador estão associados à institucionalização do paciente.

Pessoas com deficiência cognitiva, além de todas as suas limitações, também têm problemas de saúde oral mais rapidamente progressivos, incluindo úlceras linguais, hiperplasia da mucosa, estomatite subdividida, má higiene bucal, xerostomia, pior condição periodontal e mais cárie coronária e radicular (HERRERA; LOPEZ; OSORIO, 2015).

Já o câncer pode-se destacar como sendo fundamentalmente uma doença genética, cuja clonalidade, autonomia, anaplasia, invasão e metástase dos seus constituintes, são fatores de risco para a saúde desses pacientes. Além disso, compreende um grupo de cerca de 100 diferentes tipos e que, democraticamente atinge todas as faixas etárias, com a cavidade oral aparecendo junto com a pele, mama, pulmão e colo uterino, as regiões anatômicas mais prevalentemente envolvidas. A sintomatologia está diretamente associada ao sítio anatômico tamanho da lesão e grau invasivo, além da presença de metástase (MANOHARAN et al., 2016).

O termo boca, cavidade oral, refere-se aos lábios, língua, mucosa labial e bucal, gengiva, palato, região retro molar e assoalho da boca (WARNAKULASURIYA, 2010; WEATHERSPOON, 2015). O câncer de boca é uma denominação que inclui várias localizações primárias de tumor, incluídas nos códigos C00 a C08 da Classificação Internacional das Doenças (CID 10), e sua incidência global anual é estimada em cerca de 263.000 novos casos, com 127.000 mortes. (INCA, 2021).

Segundo Gupta, Jhonson e Kumar (2016) o câncer de boca é considerado uma doença multifatorial resultante da associação de elementos ambientais e genéticos, tendo o fumo, o álcool e o HPV, particularmente HPV-16 e HPV-18, como fatores considerados de risco (SIMARD; TORRE; JEMAL, 2014; ALMEIDA et al., 2011), além da qualidade nutricional dos indivíduos (JOHNSON et al., 2011; SIMARD; TORRE; JEMAL, 2014). De acordo com pesquisa descrita por Fenech e Bonassi (2011), variáveis como a idade, o gênero, dieta e estilo de vida estão relacionados com alterações presentes na mucosa oral e que são favoráveis ao desenvolvimento da patologia.

O cigarro destaca-se como o maior fator de risco para o desenvolvimento da doença, porém alguns autores descrevem que as campanhas de educação contra o tabagismo reduziram o consumo do cigarro, mas esta redução não se refletiu na incidência da doença, o que eles atribuem ao aumento do HPV (RILEY III et al., 2013; SIMARD; TORRE; JEMAL, 2014; GARBUGLIA, 2014; RETTIG; SOUZA, 2015).

A idade, a localização da lesão, bem como extensão e estadiamento tumoral impactam diretamente, de forma positiva ou negativamente, no prognóstico dos pacientes com câncer de boca (ALMEIDA et al., 2011). A diminuição da sobrevida com o aumento da idade pode estar associada à maior incidência de doenças debilitantes, às maiores sequelas pós-tratamento e 


\section{RECIMA21 - REVISTA CIENTÍFICA MULTIDISCIPLINAR ISSN 2675-6218}

DETERMINAÇÃO DA INSTABILIDADE CROMOSSÔMICA E MORTE CELULAR EM CÉLULAS DA MUCOSA BUCAL EM PACIENTES COM DEFICIÊNCIA COGNITIVA Isabela Soares Uchôa, Francisco Ariel Paz Santos Freitas, Maria do Amparo Veloso Magalhães, Suzanne Tatianne Teixeira Nascimento, Matheus Fernandes de Castro, Nelson Jorge Carvalho Batista

às complicações associadas ao envelhecimento; já o diagnóstico precoce é a medida mais eficaz para o aumento da sobrevida (CHANG et al., 2011; CHU et al., 2011).

O câncer é um processo de múltiplos estágios que resulta de um acúmulo de várias alterações genéticas. A inclusão de marcadores genéticos para avaliação do potencial mutagênico de agentes físicos, químicos e biológicos permite uma maior compreensão dos fatores de predisposição à ocorrência de neoplasias (FARIA; BRAGA, 2015). Micronúcleos são fragmentos ou cromossomas inteiros que estão fora do núcleo durante a mitose; através do seu estudo pode se avaliar os efeitos genotóxicos ambiental e ocupacional. Este teste é amplamente utilizado e é uma alternativa eficaz, simples e econômica para detectar a perda de material genético.

Atualmente, há múltiplos testes utilizados no biomonitoramento de populações humanas exposta a mutágenos, destacando-se entre eles o Teste de Micronúcleo, no qual é considerado um dos testes citogenéticos mais bem estabelecidos no campo da genética toxicológica, com validação internacional e aplicável a qualquer população celular nucleada. Este teste apresenta vantagens sobre a análise cromossômica clássica por ser um procedimento simples, eficaz e realizado a baixo custo (FREITA et al., 2014).

Objetivou-se através dessa pesquisa, avaliar a instabilidade cromossômica e morte celular em células da mucosa bucal em pacientes com deficiência cognitiva atendidos em serviço de referência odontológica a pessoa com deficiência intelectual e autismo em Teresina-PI.

\section{METODOLOGIA}

O trabalho foi realizado com base em um estudo qualitativo e quantitativo amostral que visou à coleta, e a análise de dados e material biológico coletados no Serviço de referência odontológica à pessoa com deficiência intelectual e autismo do Hospital Dirceu Arcoverde da Polícia Militar do Piauí, na cidade de Teresina, Piauí.

O estudo qualitativo trata-se de critérios subjetivos aos objetivos que buscam compreender os fenômenos sociais (educação, sociologia, psicologia, antropologia, dentre inúmeras outras áreas do conhecimento). Os dados são coletados na maioria das vezes por meio de questionários, entrevistas, grupos focais, reuniões, observação com a participação do pesquisador, dentre outras formas (MACÊDO; EVANGERLANDY, 2018)

A análise quantitativa é utilizada em pesquisas que se faz necessária a quantificação por métodos de análises estatísticas e/ou probabilísticas, com resultados por meio de gráficos quantificados, tabelas, porcentagens e/ou cálculos de erros. Já o estudo amostral ou por amostragem tem por base a representatividade real da amostra em relação à população estudada, representado assim subconjunto de sujeitos extraído de uma população por meio da caracterização do estudo, ou seja, o uso de estudo amostral tem como finalidade trabalhar com 


\section{RECIMA21 - REVISTA CIENTÍFICA MULTIDISCIPLINAR ISSN 2675-6218}

DETERMINAÇÃO DA INSTABILIDADE CROMOSSÔMICA E MORTE CELULAR EM CÉLULAS DA MUCOSA BUCAL EM PACIENTES COM DEFICIÊNCIA COGNITIVA Isabela Soares Uchôa, Francisco Ariel Paz Santos Freitas, Maria do Amparo Veloso Magalhães, Suzanne Tatianne Teixeira Nascimento, Matheus Fernandes de Castro, Nelson Jorge Carvalho Batista

uma pequena parte dos elementos que compõem o universo (MACÊDO; EVANGERLANDY, 2018).

O Serviço de Referência Odontológica à Pessoa com Deficiência Intelectual e Autismo, implantado no dia 27 de abril de 2017, atende uma média de 20 pacientes por mês, portadores de deficiência cognitiva e está localizado no Hospital Dirceu Arcoverde da Polícia Militar do Piauí, na cidade de Teresina, Piauí, que é um Hospital especializado no atendimento à Assistência MédicoHospitalar, tendo como principal finalidade prevenir doenças, promover a saúde, estimular a educação e desenvolver pesquisa. Dispõe de um quadro de profissionais responsáveis e comprometidos que estão trabalhando para o bem-estar da população Militar e Civil com a Filosofia - "Humanizando e Cuidando Bem de Sua Saúde", com uma gestão moderna pautada nos princípios de Eficiência e Eficácia. Situado na Av. Higino Cunha №. 1642, Bairro Ilhotas, com localização privilegiada na zona sul, próximo à ponte Wall Ferraz, que dá acesso às zonas leste e sudeste da cidade, sendo servido por várias linhas de transporte coletivo. Atende nas áreas de Cirurgia Geral, Cirurgia Plástica, Cirurgia Vascular. Urologia, Proctologia, Ortopedia, Ginecologia, Gastroenterologia, Clínica Geral, Oftalmologia, Odontologia, Cardiologia Clínica, Mastologia Clínica, Fonoaudiologia e Fisioterapia;

O Serviço de Odontologia atende uma clientela cada vez maior com um grau de excelência crescente. Desenvolve as seguintes atividades: dentisteria, endodontia, periodontia, exodontia, cirurgia buco-maxilo-facial (ambulatorial e cirúrgico), além de pequenas cirurgias, nas seguintes condições:

- 09 consultórios funcionando de segunda a sexta-feira das 07:00 h às 19:00 h;

- 01 consultório moderno instalado em uma área planejada em consonância com a legislação de acessibilidade e uma enfermaria com 04 leitos masculino e feminino para atendimento específico às pessoas com deficiência cognitiva, local específico onde será realizada a coleta de materiais, com total respeito à individualidade, intimidade e particularidades de cada paciente e seus familiares.

Fizeram parte da população de estudo todos os 30 indivíduos sem restrição de gênero, com registro de atendimento de maio de 2017 a janeiro de 2019, que retornaram para reavaliação oral de rotina. Um grupo controle pareado de pacientes normosistêmicos também foi abordado ( $n=$ 30), após a assinatura do Termo de Consentimento Livre e Esclarecido (TCLE).

Foi utilizada amostragem aleatória simples, que inclui todos os indivíduos com o registro completo com relação às variáveis em questão, no período em estudo delimitado. Utilizou-se como critério de exclusão a recusa do responsável pelo paciente em participar da pesquisa se negando a assinar o TCLE. O questionário tem como objetivo coletar informações básicas para que ajudem na interpretação dos dados analisados, fundamentando ainda mais a análise das alterações cromossômicas. 


\section{RECIMA21 - REVISTA CIENTÍFICA MULTIDISCIPLINAR ISSN 2675-6218}

DETERMINAÇÃO DA INSTABILIDADE CROMOSSÔMICA E MORTE CELULAR EM CÉLULAS DA MUCOSA BUCAL EM PACIENTES COM DEFICIÉNCIA COGNITIVA Isabela Soares Uchôa, Francisco Ariel Paz Santos Freitas, Maria do Amparo Veloso Magalhães, Suzanne Tatianne Teixeira Nascimento, Matheus Fernandes de Castro, Nelson Jorge Carvalho Batista

Segundo Appolinário (2012), a amostra aleatória simples se refere ao estudo em que a amostra é selecionada de maneira que a escolha de um membro da população não afete a probabilidade de seleção de qualquer outro membro. Cada membro da população tem chances iguais de ser selecionado para a amostra.

Para coleta de dados utilizou-se um questionário adaptado de Carrano e Natarajam, (1988), detalhado e organizado, com perguntas abertas e fechadas, que foi respondido pelo responsável do paciente com deficiência cognitiva, um questionário em que foi abordado detalhes da patologia e um questionário que buscou dados sobre as percepções do responsável e/ou cuidador responsável pelo deficiente cognitivo, atendendo aos objetivos propostos na pesquisa. As perguntas tiveram por base a verificação dos hábitos e rotina dos indivíduos entrevistados onde foi possível analisar as condições de vulnerabilidade e exposição aos riscos de formação de micronúcleos em célula da mucosa oral.

Segundo Gil (2010), a técnica de utilização de coleta de dados por questionário tem como base a elaboração de questões previamente elaboradas. Para Appolinário (2012), as perguntas abertas são aquelas nas quais o respondente pode escrever livremente, dentro do espaço pautado, enquanto que as perguntas fechadas oferecem algumas opções restritas de respostas possíveis.

Todas as amostras e informações pessoais foram coletadas durante os meses de fevereiro a julho de 2019, no espaço reservado para atendimento dos portadores de deficiência cognitiva, em consultório especifico e enfermaria especializada no Hospital Dirceu Arcoverde da Polícia Militar do Piauí.

As amostras de mucosa bucal foram coletadas com auxílio de escova citológica (citobrush) e foram colocadas em um tubo eppendorf seguindo-se recomendações do protocolo descrito por Thomas et al. (2009). Codificaram-se as lâminas analisadas em testes cego e os dados computados em planilhas. Os parâmetros considerados foram aqueles propostos por Thomas et al. (2009), exemplificados na Figura 1. 


\section{RECIMA21 - REVISTA CIENTÍFICA MULTIDISCIPLINAR ISSN 2675-6218}

DETERMINAÇÃO DA INSTABILIDADE CROMOSSÔMICA E MORTE CELULAR EM CÉLULAS DA MUCOSA BUCAL EM PACIENTES COM DEFICIÊNCIA COGNITIVA Isabela Soares Uchôa, Francisco Ariel Paz Santos Freitas, Maria do Amparo Veloso Magalhães,
Suzanne Tatianne Teixeira Nascimento, Matheus Fernandes de Castro, Nelson Jorge Carvalho Batista

Figura 1. Células da mucosa oral visualizadas por microscopia

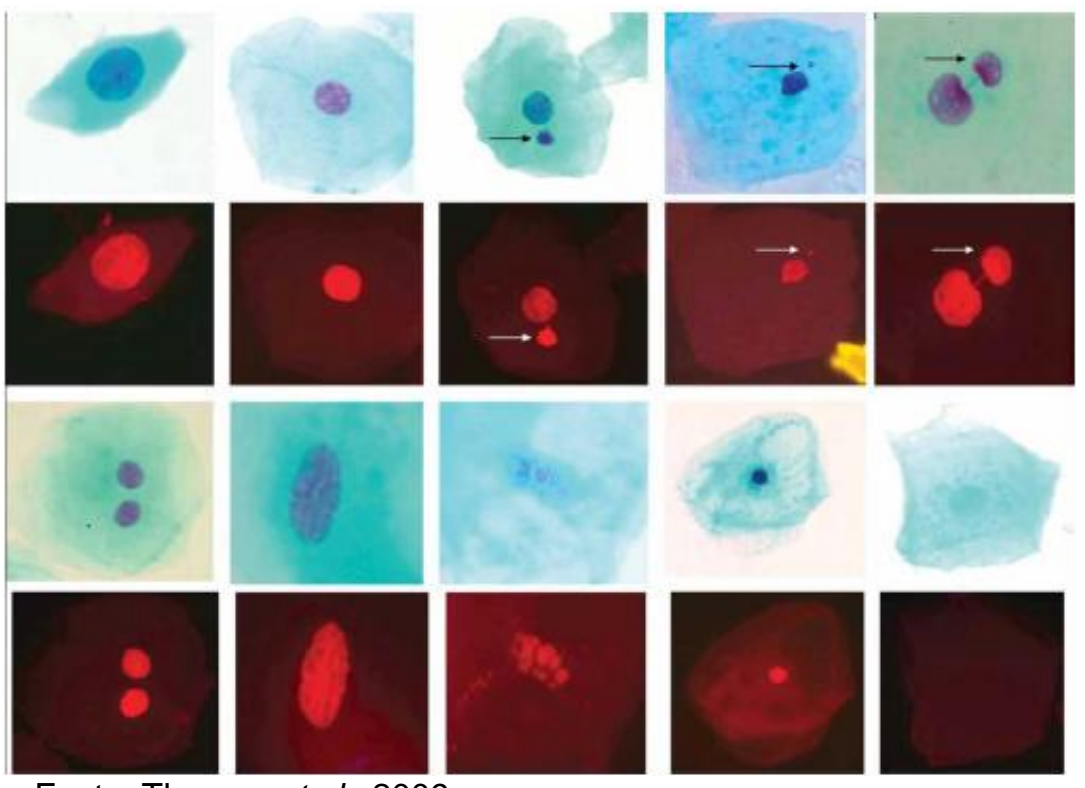

Fonte: Thomas et al., 2009

A coleta do material biológico foi realizada por meio de uma escova citológica (cytobrush) retirando-se a amostra do epitélio jugal direito e esquerdo, após um enxágue bucal realizado com água com a função de retirar material bruto que pudesse constituir um artefato no momento da análise microscópica (THOMAS et al., 2009). As células de todos os indivíduos da amostra foram coletadas de sítios da mucosa oral, ausente de ulcerações e outras lesões visíveis. Foram excluídos os indivíduos que apresentaram doenças bucais visíveis a fim de não comprometer os resultados do estudo e evitar riscos possíveis de agravamentos das lesões preexistentes. As amostras coletadas foram depositadas e transportadas em tubos eppendorf de $15 \mathrm{~mL}$ contendo a solução tampão Tris-HCl pH 7,0 (0,01 M Tris-HCl (Merck), 0,1 M EDTA tetrassódico (Vetec), 0,02 $\mathrm{M}$ cloreto (Merck)) e enviadas para o laboratório, onde foram processadas. As amostras foram centrifugadas por 10 minutos a $1000 \mathrm{rpm}$ por três vezes, desprezando-se o sobrenadante e completando o volume de $5 \mathrm{ml}$ com o tampão Tris-HCl. Este procedimento auxiliou na remoção de bactérias e restos alimentares que poderiam prejudicar a contagem. Cerca de $40 \mu l$ da solução contendo as amostras foram transferidas para lâminas, secas ao ar livre por 24 horas ou mais, e posteriormente, fixadas em metanol a $80 \%$.

A metodologia escolhida para a coloração foi a de Feulgen-Fast Green, que consiste na utilização do corante Schiff e contra-coloração de Fast-Green. Para a sua realização, as lâminas foram imersas em uma solução de $\mathrm{HCl}$ e $1 \mathrm{~N}$ por 10 minutos a uma temperatura de $60^{\circ} \mathrm{C}$, lavadas com água e coradas no Schiff por no mínimo uma hora e 30 minutos no escuro, e em seguida, lavadas com água. Após, as lâminas foram coradas com a solução alcoólica de Fast-Green a 


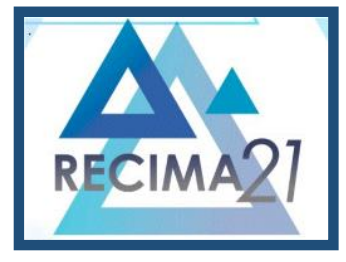

\section{RECIMA21 - REVISTA CIENTÍFICA MULTIDISCIPLINAR ISSN 2675-6218}

DETERMINAÇÃO DA INSTABILIDADE CROMOSSÔMICA E MORTE CELULAR EM CÉLULAS DA MUCOSA BUCAL EM PACIENTES COM DEFICIÊNCIA COGNITIVA Isabela Soares Uchôa, Francisco Ariel Paz Santos Freitas, Maria do Amparo Veloso Magalhães, Suzanne Tatianne Teixeira Nascimento, Matheus Fernandes de Castro, Nelson Jorge Carvalho Batista

0,1\% por 10 segundos e lavadas em água corrente para posterior leitura. A escolha pelo sítio da coleta de amostra de células de origem da mucosa jugal deve-se ao fato da facilidade da coleta do material, ser uma técnica não invasiva e de simples execução, onde a identificação de micronúcleos já se mostrou de grande eficiência (FENECH et al., 2013).

Foram avaliadas as células e os micronúcleos de acordo com os seguintes critérios sugeridos por Thomas et al. (2009) e colaboradores: (a) perímetro redondo que sugere uma membrana: (b) ter menos que um terço do diâmetro do núcleo principal, mas grande o suficiente para discernir a forma e a cor; (c) Feulgen positivo, coloração rosa em um campo iluminado; (d) intensidade de coloração similar ao do núcleo; (e) textura similar ao do núcleo; (f) mesmo plano focal que o núcleo; (g) ausência de sobreposição, ou ligação com o núcleo. Além disso, outras alterações nucleares que indica, danos no DNA foram analisadas e contabilizadas: (a) broto nuclear (brokenegg); (b) célula binucleada; (c) núcleo fragmentado. A análise consistiu de uma avaliação de no mínimo 100 células de cada duplicata da amostra. Na análise, foram consideradas variáveis como bebidas, medicamentos, idade, sexo e fumo como potenciais fatores de interferência.

A pesquisa foi realizada mediante autorização por escrito do representante legal da Instituição supracitada, em consonância com a Portaria do Ministério da Saúde, Conselho Nacional de Ética em Pesquisa, que regulamenta as questões operacionais e éticas dos trabalhos científicos envolvendo seres humanos. O presente estudo foi submetido a análise pelo Comitê de Ética em Pesquisa do Centro Universitário Santo Agostinho (CEP/UNIFSA), com o que determina as Resoluções no 466/2012 e 510/2016 do Conselho Nacional Saúde do Ministério da Saúde (CNS/MS).

Toda a pesquisa baseou-se na resolução 466/12 e 510/2016, onde toda a coleta de dados foi realizada de forma a não expor os sujeitos na qual foram decodificados em números e sua identidade mantida em sigilo, respeitando o direito de livre escolha, buscando diminuir os desconfortos com utilização de material adequada e ambiente privado, sendo que foi realizada após a confirmação voluntaria, onde o pesquisador respeitou as normas e rotinas vigentes.

Após a coleta de dados, os mesmos foram tabulados em planilhas do programa de computador Microsoft Excel em seguida analisados, no software BioStat 2008 v. 5. Primeiramente, realizou-se o cálculo das frequências (análise univariadas) totais de micronúcleos e outras alterações citogenéticas nos grupos estudados. Foram realizados cálculos para observar a distribuição da instabilidade cromossômica de micronúcleos e células basais da mucosa bucal e outras anormalidades nucleares dentro deste grupo e comparadas médias de frequência relativa de variáveis como fumo, bebida, horas de trabalho, sexo, etc. Obtidas no questionário segundo micronúcleo, brokenegg, células binucleadas e núcleos fragmentado, almejando-se o intervalo de confiança de $95 \%$. 


\section{RECIMA21 - REVISTA CIENTÍFICA MULTIDISCIPLINAR ISSN 2675-6218}

DETERMINAÇÃO DA INSTABILIDADE CROMOSSÔMICA E MORTE CELULAR EM CÉLULAS DA MUCOSA BUCAL EM PACIENTES COM DEFICIÊNCIA COGNITIVA Isabela Soares Uchôa, Francisco Ariel Paz Santos Freitas, Maria do Amparo Veloso Magalhães, Suzanne Tatianne Teixeira Nascimento, Matheus Fernandes de Castro, Nelson Jorge Carvalho Batista

Após a conclusão das pesquisas e análise dos dados, os participantes tomarão conhecimento dos resultados através de uma exposição do relatório final, apresentado em palestra pública a ser realizada no auditório do HPMPI, publicações científicas e nos encontros acadêmicos.

\section{RESULTADOS E DISCUSSÃO}

O teste de micronúcleos foi aplicado na amostra da mucosa oral de 30 indivíduos sem restrição de gênero, que retornaram para reavaliação oral de rotina no HPMPI. Um grupo controle pareado de pacientes normosistêmicos também foi abordado $(n=30)$, após a assinatura do Termo de Consentimento Livre e Esclarecido (TCLE).

No quadro 1 constam os dados obtidos em relação a doença cognitiva, gênero e idade de cada indivíduo que compôs a amostra. Vale ressaltar, que os pacientes foram numerados de 1 a 30 , conforme a ordem em que a coleta da mucosa oral foi realizada.

Essas informações foram produzidas para categorizar quais doenças de base os pacientes apresentavam, além de estipular a correlação entre a presença dessas patologias com a idade e gênero dos pacientes, já que não houve restrições quanto a isso.

Quadro 1 - Número dos pacientes e deficiência cognitiva. Teresina, Piauí, 2019(n=30)

\begin{tabular}{|c|c|c|c|}
\hline $\begin{array}{c}\text { No } \\
\text { Indivíduo }\end{array}$ & $\begin{array}{c}\text { Idade } \\
\text { (anos) }\end{array}$ & Sexo & Deficiência Cognitiva \\
\hline 1 & 21 & $\mathrm{M}$ & TEA \\
\hline 2 & 29 & $\mathrm{~F}$ & Deficiência Mental Severa \\
\hline 3 & 12 & $\mathrm{M}$ & Paralisia Cerebral \\
\hline 4 & 36 & $\mathrm{~F}$ & Deficiência Intelectual \\
\hline 5 & 37 & $\mathrm{M}$ & Deficiência Psicomotora \\
\hline 6 & 15 & $\mathrm{M}$ & TEA \\
\hline 7 & 27 & $\mathrm{M}$ & TEA/Deficiência Visual \\
\hline 8 & 28 & $\mathrm{M}$ & Paralisia Cerebral \\
\hline 9 & 28 & $\mathrm{M}$ & Síndrome de Down \\
\hline 10 & 17 & $\mathrm{~F}$ & Paralisia Cerebral \\
\hline 11 & 29 & $\mathrm{M}$ & Retardo Mental Grave \\
\hline 12 & 24 & $\mathrm{~F}$ & AVC Hemorrágico \\
\hline 13 & 15 & $\mathrm{M}$ & Paralisia Cerebral/TEA \\
\hline 14 & 31 & $\mathrm{M}$ & Deficiência Neurológica com \\
atraso
\end{tabular}

RECIMA21 - Ciências Exatas e da Terra, Sociais, da Saúde, Humanas e Engenharia/Tecnologia 
RECIMA21 - REVISTA CIENTÍFICA MULTIDISCIPLINAR ISSN 2675-6218

DETERMINAÇÃO DA INSTABILIDADE CROMOSSÔMICA E MORTE CELULAR EM CÉLULAS DA MUCOSA BUCAL EM PACIENTES COM DEFICIÊNCIA COGNITIVA Isabela Soares Uchôa, Francisco Ariel Paz Santos Freitas, Maria do Amparo Veloso Magalhães, Suzanne Tatianne Teixeira Nascimento, Matheus Fernandes de Castro, Nelson Jorge Carvalho Batista

\begin{tabular}{|c|c|c|c|}
\hline 21 & 31 & M & Paralisia Cerebral \\
\hline 22 & 41 & F & $\begin{array}{c}\text { Paralisia Cerebral/Paralisia } \\
\text { Hemiplégica Direita }\end{array}$ \\
\hline 23 & 27 & M & Síndrome de Down \\
\hline 24 & 24 & M & Hidrocefalia \\
\hline 25 & 35 & F & Deficiência Mental/Física \\
\hline 26 & 34 & F & Síndrome de Down \\
\hline 27 & - & F & $\begin{array}{c}\text { Hidrocefalia/Deficiência } \\
\text { Intelectual }\end{array}$ \\
\hline 28 & - & F & Deficiência Intelectual \\
\hline 29 & 29 & F & Microcefalia \\
\hline 30 & 28 & F & Síndrome de Down \\
\hline
\end{tabular}

Fonte: Autores da pesquisa

Conforme o quadro 1 , existe uma variedade de doenças neurodegenerativas diagnosticadas nos pacientes que compõem a amostra. Porém, é possível destacar algumas que aparecem com mais frequência, dentre elas, 7 indivíduos tem Síndrome de Down, 6 apresentam paralisia cerebral (PC), 5 possuem transtorno do espectro autista (TEA) e 4 aparecem com deficiência intelectual.

Além disso, foi possível destacar que dos 30 pacientes, 16 são do sexo masculino e 24 do sexo feminino, dessa forma, aleatoriamente a amostra foi composta por um número maior de mulheres, o que significa que essas doenças se apresentaram com mais frequência nesse grupo. No tocante a idade, apenas um paciente apresentou idade entre 0 - 10 anos, 5 pacientes estavam na faixa de 11-20 anos, 9 pacientes apresentaram idade igual ou superior a 31 anos, mas a faixa etária mais frequente foi de 21-30 anos com 13 indivíduos. Vale frisar que não se obteve a idade dos pacientes que correspondem a amostra número 27 e 28, algumas entrevistas foram prejudicadas por conta do cenário marcado pela pandemia do covid-19, e os prontuários de atendimento desses indivíduos estavam incompletos.

De acordo com a bibliografia levantada por Uchôa e Magalhães (2019), os trabalhos que envolvem a aplicação do teste de micronúcleos, são diversos e podem ser aplicados em pacientes que apresentam patologias variadas, como biomarcador capaz de predizer os riscos de câncer. Porém, baseado nesse mesmo estudo, constatou-se que a quantidade de artigos encontrados com o teste aplicado em pacientes com doenças neurodegenerativas foi muito aquém comparada com pacientes que apresentavam outros tipos de patologias, ou que se encontravam expostos a algum material que causasse danos genéticos, tornando-se um vasto campo para ampliação dos estudos.

Realizou-se o teste de MN nos pacientes com doenças cognitivas e a tabela 1 ilustra os resultados da frequência de micronúcleos e outras alterações celulares que foram encontradas em comparação ao grupo controle da pesquisa. 


\section{RECIMA21 - REVISTA CIENTÍFICA MULTIDISCIPLINAR ISSN 2675-6218}

DETERMINAÇÃO DA INSTABILIDADE CROMOSSÔMICA E MORTE CELULAR EM CÉLULAS DA MUCOSA BUCAL EM PACIENTES COM DEFICIÊNCIA COGNITIVA Isabela Soares Uchôa, Francisco Ariel Paz Santos Freitas, Maria do Amparo Veloso Magalhães,
Suzanne Tatianne Teixeira Nascimento, Matheus Fernandes de Castro, Nelson Jorge Carvalho Batista

Tabela 1 - Frequência de Micronúcleo, Pontes Nucleoplasmáticas, Buds Nucleares, Índice de Divisão Nuclear, Células Binucleadas, Cromatina Condensada, Cariorrexe, Cariólise e Picnose (média \pm desvio padrão) no Grupo Controle e em Pacientes com Deficiência Cognitiva. Teresina, Piauí, 2019.

\begin{tabular}{lccc}
\hline \multicolumn{1}{c}{ Parâmetros } & \multicolumn{2}{c}{ Grupos } & \\
\cline { 2 - 3 } & $\begin{array}{c}\text { Controle } \\
(\boldsymbol{n}=\mathbf{3 0})\end{array}$ & $\begin{array}{c}\text { Pacientes com } \\
\text { Deficiência Cognitiva } \\
(\boldsymbol{n}=\mathbf{3 0})\end{array}$ & $\boldsymbol{p}$ \\
\hline Micronúcleo & $8.6 \pm 2.8$ & $12.2 \pm 3.6$ & 0.016 \\
Pontes & $0.8 \pm 0.4$ & $1.8 \pm 0.8$ & 0.004 \\
Nucleoplasmáticas & $1.7 \pm 1.0$ & $3.0 \pm 1.6$ & 0.008 \\
Buds Nucleares & $1.8 \pm 0.8$ & $2.2 \pm 1.0$ & 0.160 \\
IDN & $14.0 \pm 4.0$ & $30.0 \pm 15.8$ & 0.008 \\
Células Binucleadas & $38.0 \pm 24.2$ & $68.2 \pm 28.2$ & 0.008 \\
Cromatina & $18.0 \pm 16.2$ & $34.3 \pm 18.7$ & 0.060 \\
Condensada & $180.2 \pm 124.2$ & $292.6 \pm 143.4$ & 0.090 \\
Cariorrexe & $2.8 \pm 1.2$ & $6.2 \pm 1.6$ & 0.020 \\
Cariólise & & & \\
Picnose & & & \\
\hline
\end{tabular}

IDN= índice de divisão do nuclear em 1000 células por sujeito, $n=$ número de sujeitos. Teste t de Student ou teste $\mathrm{U}$ de Mann Whitney.

Fonte: BioStat 2008 v. 5

Após a coleta da mucosa oral dos pacientes, foi realizada a coloração das lâminas e a contagem de 1000 células por sujeito, os resultados obtidos levaram em consideração os parâmetros sugeridos por Thomas et al. (2009) que estão associados aos tipos de alterações celulares que podem ser encontrados em indivíduos que se expõem a algum fator genotóxico ou que acarrete mudanças no DNA celular. 


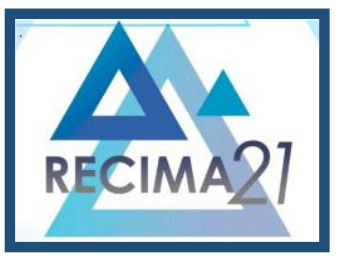

\section{RECIMA21 - REVISTA CIENTÍFICA MULTIDISCIPLINAR ISSN 2675-6218}

DETERMINAÇÃO DA INSTABILIDADE CROMOSSÔMICA E MORTE CELULAR EM CÉLULAS DA MUCOSA BUCAL EM PACIENTES COM DEFICIÊNCIA COGNITIVA Isabela Soares Uchôa, Francisco Ariel Paz Santos Freitas, Maria do Amparo Veloso Magalhães, Suzanne Tatianne Teixeira Nascimento, Matheus Fernandes de Castro, Nelson Jorge Carvalho Batista

Figura 2: Alterações celulares encontradas durante a análise das lâminas.
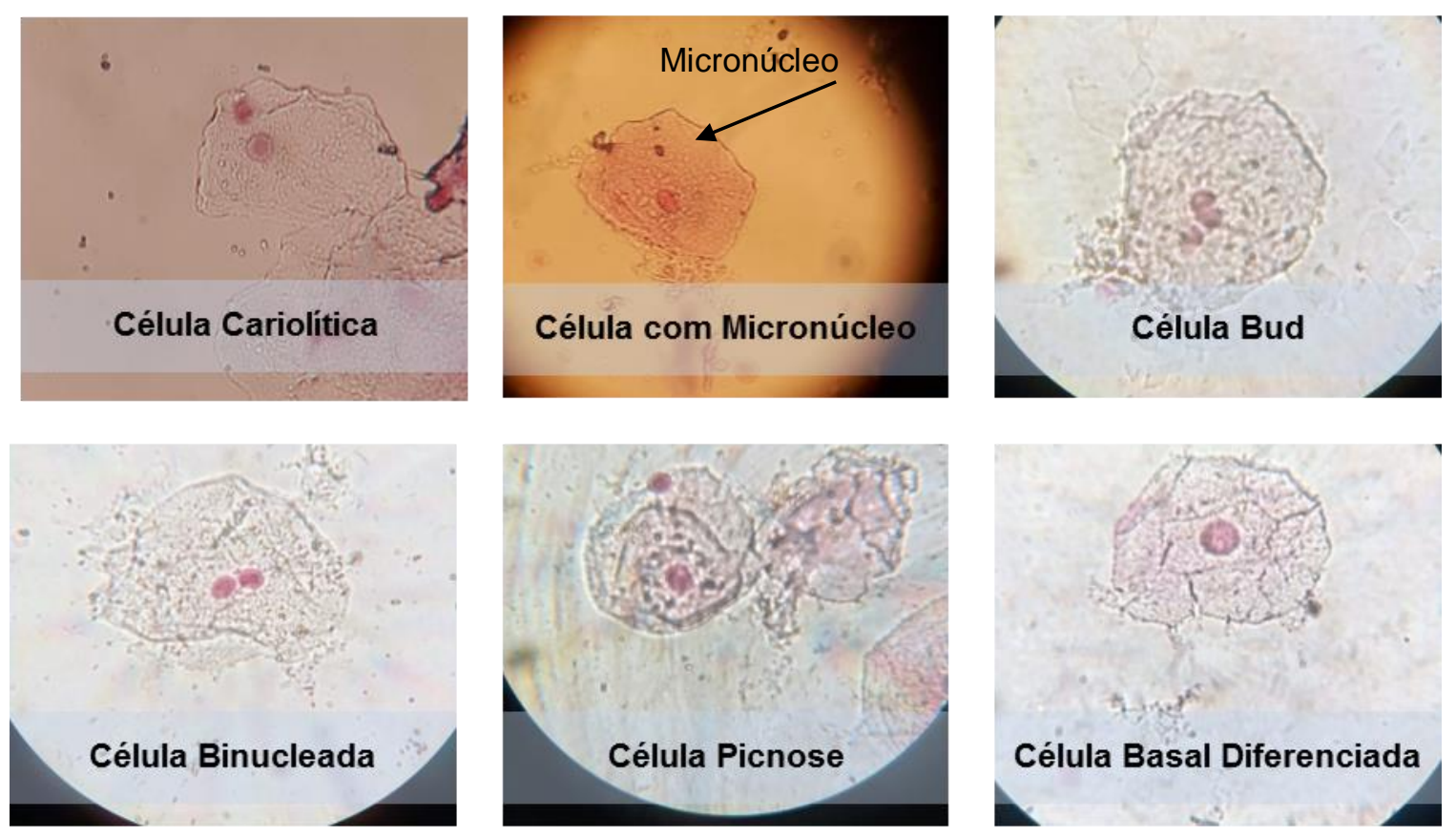

Fonte: Autores da pesquisa

Thomas et al. (2009) seu estudo, descreveram cada um dos parâmetros apresentados. Segundo eles, as células micronucleadas são caracterizadas pela presença de um núcleo principal e um ou mais núcleos menores, que são estruturas que se fragmentam durante a divisão celular. Já as células Bud são assim denominadas por possuírem botões nucleares, formações que estão bem próximos ao núcleo principal, podendo estar relacionada a eliminação do DNA amplificado. Outra alteração que pode ser encontrada são as células binucledas, que contêm dois núcleos principais em vez de um, originadas de falhas provenientes da citocinese.

Ainda segundo Thomas et al. (2009), as células com cromatina condensada mostram um aproximadamente padrão nuclear estriado em que a cromatina está se agregando em algumas regiões do núcleo enquanto é perdidos em outras áreas. Já as células cariorréticas têm núcleos que são caracterizados por mais extensos agregação de cromatina nuclear em relação a células de cromatina condensada. Eles têm um padrão nuclear densamente pontilhado indicativo de fragmentação nuclear levando ao eventual desintegração do núcleo. As células picnóticas por sua vez são caracterizadas por um pequeno encolhimento do núcleo, com uma alta densidade de material nuclear e acredita-se que essas células podem estar passando por uma forma única de morte celular. Por fim ss células cariolíticas são células em que o núcleo é completamente esgotado de DNA e é aparente como um fantasma. Portanto, essas células parecem não ter núcleo e representam um estágio muito tardio no processo de morte celular. 


\section{RECIMA21 - REVISTA CIENTÍFICA MULTIDISCIPLINAR ISSN 2675-6218}

DETERMINAÇÃO DA INSTABILIDADE CROMOSSÔMICA E MORTE CELULAR EM CÉLULAS DA MUCOSA BUCAL EM PACIENTES COM DEFICIÊNCIA COGNITIVA Isabela Soares Uchôa, Francisco Ariel Paz Santos Freitas, Maria do Amparo Veloso Magalhães, Suzanne Tatianne Teixeira Nascimento, Matheus Fernandes de Castro, Nelson Jorge Carvalho Batista

O teste de hipótese descrito na tabela 1 , levou em consideração o valor $\alpha=5 \%$, como nível de significância. Dessa forma, o P-valor sendo menor que $5 \%$ ou 0.05 , pode-se considerar como falsa a hipótese nula, de que pacientes com deficiência cognitiva não apresentam relação com a formação de micronúcleos em células basais da mucosa oral, devendo-se considerar a hipótese alternativa em que essa relação pode existir.

Os resultados demonstraram que a frequência de micronúcleos, bem como das demais alterações celulares foi maior em pacientes com deficiência cognitiva do que no grupo controle. Observou-se que o P-valor do parâmetro micronúcleos foi igual a 0.016, sendo menor que o nível de significância estabelcido de 0.05 . Isso também aconteceu nos parâmetros de pontes nucleoplamáticas $(p=0.004)$, buds nucleares $(p=0.008)$, células binucleadas $(p=0.008)$, cromatina condensada $(p=0.008)$ e células picnoses $(p=0.020)$. Dessa forma, foi possivél inferir que provavelmente exista uma relação entre a doença de base associada à deficiência cognitiva e a formação de micronúcleos em células basais da mucosa oral, bem como a presença de outras alterações celulares.

O estudo também buscou descrever o perfil dos pacientes atendidos, com o objetivo de estabelecer uma relação entre a frequência basal de micronúcleos presentes em células da mucosa bucal de pacientes com deficiência cognitiva a as variáveis nutricionais, genéticas e de exposição ambiental.

Quadro 2 - Perfil dos pacientes $(n=30)$.

\begin{tabular}{|c|c|c|c|c|c|c|}
\hline No & $\begin{array}{c}\text { Exposição } \\
\text { a } \\
\text { substâncias } \\
\text { químicas }\end{array}$ & $\begin{array}{c}\text { Histórico de } \\
\text { doenças }\end{array}$ & $\begin{array}{c}\text { Histórico } \\
\text { de } \\
\text { câncer } \\
\text { na } \\
\text { família }\end{array}$ & $\begin{array}{c}\text { Restrição } \\
\text { alimentar }\end{array}$ & $\begin{array}{c}\text { Comportamento } \\
\text { social }\end{array}$ & $\begin{array}{c}\text { Medicações em } \\
\text { uso }\end{array}$ \\
\hline 01 & Não & Não & Não & Não & Hiperativo & $\begin{array}{c}\text { Longatil, fernegan } \\
\text { levozin }\end{array}$ \\
\hline 02 & Não & Não & Não & Não & Instável & $\begin{array}{c}\text { Longactil, } \\
\text { risperidona } \\
\text { fernegan }\end{array}$ \\
\hline 03 & Não & Não & Não & $\begin{array}{c}\text { Carne de } \\
\text { porco }\end{array}$ & Agressivo & \begin{tabular}{c} 
Neuleptil \\
\hline 04
\end{tabular} \\
\hline Não & Não & Não & Não & Assustado & $\begin{array}{c}\text { Clorpromazina, } \\
\text { epilenil com } \\
\text { valproico, halo 5 } \\
\text { mg }\end{array}$ \\
\hline 05 & Não & Convulsão & Não & Não & Tranquilo & $\begin{array}{c}\text { Levozine, } \\
\text { carbamazepina, } \\
\text { risperidona }\end{array}$ \\
\hline 06 & Não & Não & Não & Não & Assustado & $\begin{array}{c}\text { Neuleptil, } \\
\text { olanzapina, } \\
\text { duloxetina. }\end{array}$ \\
\hline 07 & Não & Não & Não & Não & Tranquilo & Não informado \\
\hline 08 & Não & Não & Não & Não & Tranquilo & $\begin{array}{c}\text { Clonazepam, } \\
\text { depakene xarope }\end{array}$ \\
\hline
\end{tabular}




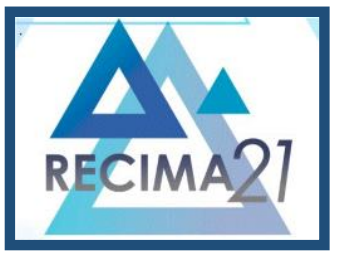

\section{RECIMA21 - REVISTA CIENTÍFICA MULTIDISCIPLINAR ISSN 2675-6218}

DETERMINAÇÃO DA INSTABILIDADE CROMOSSÔMICA E MORTE CELULAR EM CÉLULAS DA MUCOSA BUCAL EM PACIENTES COM DEFICIÊNCIA COGNITIVA Isabela Soares Uchôa, Francisco Ariel Paz Santos Freitas, Maria do Amparo Veloso Magalhães, Suzanne Tatianne Teixeira Nascimento, Matheus Fernandes de Castro, Nelson Jorge Carvalho Batista

\begin{tabular}{|c|c|c|c|c|c|c|}
\hline 09 & Não & Não & Não & Não & Tranquilo & Não informado \\
\hline 10 & Não & Não & Não & Não & Tranquilo & Carbamazepina \\
\hline 11 & Não & Não & $\operatorname{Sim}$ & Não & Assustado & $\begin{array}{c}\text { Haldol, } \\
\text { prometazina }\end{array}$ \\
\hline 12 & Não & $\begin{array}{l}\text { Rigidez } \\
\text { muscular }\end{array}$ & Não & Não & Tranquilo & Oxcarbamazepina \\
\hline 13 & Não & $\begin{array}{c}\text { Anemia, } \\
\text { deficiência } \\
\text { motora }\end{array}$ & Sim & Não & Agitado & $\begin{array}{l}\text { Não faz uso de } \\
\text { medicamentos }\end{array}$ \\
\hline 14 & Não & Não & Sim & Não & Tranquilo & Tâmisa 30 \\
\hline 15 & Não & Não & Não & Não & Tranquilo & $\begin{array}{c}\text { Carbanazepina, } \\
\text { olanzapina, } \\
\text { longatil. }\end{array}$ \\
\hline 16 & Não & Não & Não & Não & Tranquilo & Não informado \\
\hline 17 & Não & Não & Sim & Não & Tranquilo & Não informado \\
\hline 18 & Não & Não & Não & Não & Tranquilo & Não informado \\
\hline 19 & Não & Não & Sim & Não & Agressivo & $\begin{array}{l}\text { Depakote, } \\
\text { risperidona }\end{array}$ \\
\hline 20 & Não & Hipertireoidismo & Sim & Não & Tranquilo & Não informado \\
\hline 21 & Não & Não & Não & Não & Tranquilo & Não informado \\
\hline 22 & Não & $\begin{array}{c}\text { Paralisa à } \\
\text { direita }\end{array}$ & Não & Não & Tranquilo & $\begin{array}{l}\text { Risperidona, } \\
\text { amitriptilina }\end{array}$ \\
\hline 23 & Não & Não & Sim & Não & Tranquilo & Não informado \\
\hline 24 & Não & Não & Não & Não & Tranquilo & Não informado \\
\hline 25 & Não & Não & Não & Não & Tranquilo & $\begin{array}{c}\text { Fenobarbital, } \\
\text { carbamazepina }\end{array}$ \\
\hline 26 & Não & Não & Sim & Não & Tranquilo & Não informado \\
\hline 27 & Não & Não & Não & Não & Tranquilo & Não informado \\
\hline 28 & Não & Não & Não & Não & Tranquilo & Não informado \\
\hline 29 & Não & Não & Não & Não & Tranquilo & Gardenal \\
\hline 30 & Não & Hipertireoidismo & Sim & Não & Tranquilo & Não informado \\
\hline
\end{tabular}

Fonte: Autores da pesquisa

O estudo elaborado por Freitas, Uchôa e Magalhães (2020), abordou que vários são os parâmetros biológicos que podem ser alterados como consequência da interação entre um agente externo (químico, físico ou biológico) e o organismo. Entretanto, a determinação quantitativa dos parâmetros usados como indicadores biológicos de exposição, também chamados de biomarcadores, só é possível se existir correlação com a intensidade da exposição e/ou o efeito biológico decorrente da substância em questão.

O papel da dieta na incidência de todos os cânceres epiteliais tem sido destacado em muitos estudos, além do consumo de frutas, vegetais e quantidades moderadas de carnes, podem conferir proteção para o câncer bucal, independente de fatores de risco reconhecidos, como 0 fumo e o consumo alcoólico. Assim, resultados de estudos epidemiológicos demonstram que o consumo de frutas e vegetais está sendo associado à diminuição do risco de câncer. O baixo consumo de frutas e vegetais predispõe o aparecimento do câncer bucal, à medida que pode reduzir a imunidade, desencadear processos de ceratinização excessiva e aumentar os radicais livres contribuindo para a ativação de oncogenes (LEITE; SILVA; MELO, 2005). 


\section{RECIMA21 - REVISTA CIENTÍFICA MULTIDISCIPLINAR ISSN 2675-6218}

DETERMINAÇÃO DA INSTABILIDADE CROMOSSÔMICA E MORTE CELULAR EM CÉLULAS DA MUCOSA BUCAL EM PACIENTES COM DEFICIÊNCIA COGNITIVA Isabela Soares Uchôa, Francisco Ariel Paz Santos Freitas, Maria do Amparo Veloso Magalhães, Suzanne Tatianne Teixeira Nascimento, Matheus Fernandes de Castro, Nelson Jorge Carvalho Batista

Dessa forma, os resultados do quadro 2 sugerem que os pacientes não se expõem ou fazem uso de substâncias como álcool ou fumo, a única fonte de exposição regular estaria associada às medicações de uso contínuo presente na vida da maioria. Fora isso, os pacientes apresentaram hábitos alimentares variados, consumindo todos os tipos de alimentos, incluindo frutas e verduras em sua dieta, apenas o paciente da amostra 03 possui restrição alimentar a carne de porco. Com relação a variável genética apenas 9 dos 30 indivíduos possuem histórico de câncer na família e apenas 6 dos 30 possuem histórico de outras patologias sem levar em consideração a doença de base. Por essa análise pode-se considerar que nessa amostra provavelmente o aumento da frequência de $\mathrm{MN}$ está mais relacionada com a deficiência cognitiva dos pacientes do que com as variáveis de exposição ambiental, histórico familiar ou nutricional.

Muitos pacientes apresentam comportamento social tranquilo, decorrente do uso de medicações que controlam as alterações de humor. Mesmo assim, é possível que haja mudanças nesse comportamento, verificou-se que os indivíduos se apresentaram instáveis, agressivos, hiperativos e assustados, durante a coleta de mucosa e da aplicação do questionário aos seus responsáveis, o que dificultou bastante o processo.

O quadro 3 foi produzido baseado nas principais características dos cuidadores de pacientes com deficiência cognitiva, abordando as repercussões que os cuidados às pessoas com limitações intelectuais e físicas podem trazer para a vida de quem cuida.

Quadro 3 - Perfil dos cuidadores $(n=30)$.

\begin{tabular}{|c|c|c|c|c|}
\hline № & Vínculo & $\begin{array}{l}\text { Alguém que auxilia } \\
\text { nos cuidados }\end{array}$ & $\begin{array}{c}\text { Tempo de que cuida } \\
\text { do paciente }\end{array}$ & Alterações na vida pessoal \\
\hline 01 & Mãe & A família & Desde o nascimento & Nenhuma \\
\hline 02 & Pai & A família & Desde o nascimento & Nenhuma \\
\hline 03 & Mãe & Avó materna & Desde o nascimento & Nenhuma \\
\hline 04 & Mãe & Irmão & Desde o nascimento & $\begin{array}{l}\text { Atrapalha no momento que } \\
\text { precisa acalmar o paciente. }\end{array}$ \\
\hline 05 & Mãe & Ninguém & Desde o nascimento & Nenhuma \\
\hline 06 & Mãe & Avó materna & Desde o nascimento & Dificulta a procura de emprego \\
\hline 07 & Mãe & A família & Desde o nascimento & Nenhuma \\
\hline 08 & Pai & Mãe & Desde o nascimento & Nenhuma \\
\hline 09 & Mãe & Ninguém & Desde o nascimento & Nenhuma \\
\hline 10 & Mãe & A família & Desde o nascimento & $\begin{array}{c}\text { Deixa de realizar atividades } \\
\text { pessoais }\end{array}$ \\
\hline 11 & Pai & Mãe & Desde o nascimento & Não interfere \\
\hline 12 & Mãe & Ninguém & Desde o nascimento & $\begin{array}{l}\text { Interfere devido a divisão do } \\
\text { cuidado entre os filhos. }\end{array}$ \\
\hline 13 & Pai & Mãe e a madrasta & Desde o nascimento & $\begin{array}{l}\text { Interfere nos momentos de } \\
\text { passeio }\end{array}$ \\
\hline 14 & Irmão & Pais & Desde o nascimento & Disponibiliza maior aprendizado \\
\hline 15 & Mãe & Pai & Desde o nascimento & Nenhuma \\
\hline 16 & Mãe & Ninguém & Desde o nascimento & Nenhuma \\
\hline 17 & Tio & Tia & 3 anos & Traz maiores responsabilidades \\
\hline 18 & Mãe & Irmãos & Desde o nascimento & Nenhuma \\
\hline
\end{tabular}




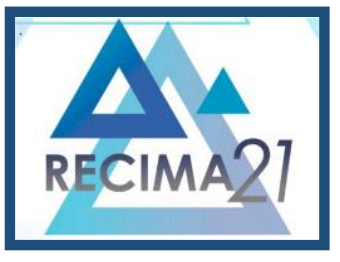

\section{RECIMA21 - REVISTA CIENTÍFICA MULTIDISCIPLINAR ISSN 2675-6218}

DETERMINAÇÃO DA INSTABILIDADE CROMOSSÔMICA E MORTE CELULAR EM CÉLULAS DA MUCOSA BUCAL EM PACIENTES COM DEFICIÊNCIA COGNITIVA Isabela Soares Uchôa, Francisco Ariel Paz Santos Freitas, Maria do Amparo Veloso Magalhães, Suzanne Tatianne Teixeira Nascimento, Matheus Fernandes de Castro, Nelson Jorge Carvalho Batista

\begin{tabular}{|c|c|c|c|c|}
\hline 19 & Mãe & Pai & Desde o nascimento & Nenhuma \\
\hline 20 & Mãe & Ninguém & Desde o nascimento & Nenhuma \\
\hline 21 & Mãe & Ninguém & Desde o nascimento & Nenhuma \\
\hline 22 & Mãe & Irmã & Desde o nascimento & $\begin{array}{c}\text { Vida restrita devido aos cuidados } \\
\text { prestados }\end{array}$ \\
\hline 23 & Mãe & Irmãs & Desde o nascimento & Nenhuma \\
\hline 24 & Mãe & Irmãos & Desde o nascimento & Nenhuma \\
\hline 25 & Mãe & Avó & Desde o nascimento & Nenhuma \\
\hline 26 & Mãe & Pai & Desde o nascimento & Nenhuma \\
\hline 27 & Mãe & A família & Desde o nascimento & Nenhuma \\
\hline 28 & Mãe & Pai & Desde o nascimento & Nenhuma \\
\hline 29 & Mãe & Ninguém & Desde o nascimento & Nenhuma \\
\hline 30 & Pai & Ninguém & Desde o nascimento & Nenhuma \\
\hline
\end{tabular}

Fonte: Autores da pesquisa.

Segundo Silva et al (2020), a condição crônica acarreta implicações no contexto familiar, podendo trazer diversas dificuldades na rotina vivenciada pela família. Nestas condições a dinâmica familiar é modificada, sendo impostas novas condições frente ao cuidado à criança. Sendo assim, geralmente a mãe toma a responsabilidade para si, como a única capaz de ter o cuidado diário e direto a essas crianças e acabam modificando seus hábitos por defenderem a ideia de que são as únicas aptas a cuidar de seus filhos/as.

Os resultados do quadro descrito acima trazem a figura materna como principal cuidadora de pacientes com deficiência cognitiva, cuidados estes que se iniciam desde o nascimento na maioria dos casos. Porém, é possível analisar que a figura do pai, dos avós, tios e irmãos também aparecem como forma de auxílio e apoio na prestação desses cuidados.

Mesmo diante de tantas dificuldades e obstáculos, a maioria dos cuidadores relataram que não há interferências nas atividades da sua vida pessoal, por conta da prestação de cuidados a esses pacientes. Porém, alguns alegaram que por conta disso, possuem dificuldades de conseguir emprego, dividir atenção com outros filhos, realizar atividades de lazer como passeios, ou até mesmo de conter as alterações de humor dos pacientes, que como visto, pode ser um grande desafio.

\section{CONCLUSÃO}

O estudo demonstra a importância da aplicação do Teste de Micronúcleo como um biomarcador confiável no diagnóstico da instabilidade genômica, pois este se constitui de uma técnica acessível pelo baixo custo, eficaz, simples e que não gera traumas aos seres humanos por não ser invasiva, sendo também usado na detecção precoce de danos genéticos.

Infere-se que a aplicação do teste em pacientes com deficiência cognitiva, por mais simples e não invasiva que fosse a técnica, apresentou-se como um grande desafio, por conta da dificuldade de interação com os pacientes, em virtude das suas limitações e oscilações de humor 


\section{RECIMA21 - REVISTA CIENTÍFICA MULTIDISCIPLINAR ISSN 2675-6218}

DETERMINAÇÃO DA INSTABILIDADE CROMOSSÔMICA E MORTE CELULAR EM CÉLULAS DA MUCOSA BUCAL EM PACIENTES COM DEFICIÊNCIA COGNITIVA Isabela Soares Uchôa, Francisco Ariel Paz Santos Freitas, Maria do Amparo Veloso Magalhães, Suzanne Tatianne Teixeira Nascimento, Matheus Fernandes de Castro, Nelson Jorge Carvalho Batista

e comportamento, mas foi fundamental para demonstrar que o aumento da frequência de micronúcleos, bem como a presença de outras alterações celulares, provavelmente esteja associada a doença de base desses pacientes, que por sua vez, gera inúmeras limitações físicas e intelectuais.

Dessa forma, o aumento da frequência de MN indica a persistência de dano cromossômico em células totalmente diferenciadas (como nas células epiteliais bucais), que por sua vez, aumenta o risco de desenvolvimento de patologias como o câncer. Nessa perspectiva, o teste de MN foi utilizado como uma ferramenta importante capaz de predizer os riscos e danos genéticos presentes nas células dos pacientes com doenças cognitivas, que além de todas as limitações causadas pela doença de base, ainda podem desenvolver câncer de boca.

O estudo também possibilitou concluir que existem inúmeros parâmetros que devem ser levados em consideração e que podem estar correlacionados ao aumento da frequência de micronúcleos, como por exemplo: variáveis de exposição, nutricionais e genéticas. Porém, na presente pesquisa, supõe-se que o fator mais relacionado ao aumento dos danos celulares presentes nas células dos pacientes com deficiência cognitiva, foi a doença de base.

No tocante aos cuidadores, percebeu-se que a principal figura detentora desses cuidados é a materna. Existem muitos obstáculos familiares e sociais que interferem nesse processo, mas foi possível analisar que o amor e carinho empregado nas falas e nas ações desses cuidadores para com seus pacientes, é maior que qualquer dificuldade.

Diante de tal importância e eficácia evidenciada pela aplicação do Teste de Micronúcleos como uma ferramenta que se mostrou efetiva na detecção de instabilidade cromossômica em pacientes com deficiência cognitiva, sugere-se que haja ampliação dos estudos referente a temática, já que existem poucas publicações na literatura relacionadas a aplicação do teste nesses pacientes.

Pesquisas como essa trazem benefícios aos pacientes, pois a partir dos dados coletados analisou-se os fatores de riscos associados à instabilidade cromossômica de micronúcleos e células basais da mucosa bucal, em pacientes com deficiência cognitiva, contribuindo para as publicações de temas referentes a patologias, proporcionando informações bem fundamentadas em relação ao tema, estimulando a pesquisa em saúde entre os mais diversos estudantes e profissionais da saúde, de modo a contribuir com aprimoramento das medidas de prevenção.

\section{REFERÊNCIAS}

ALMEIDA, Fernanda Campos Sousa et al. Fatores Prognósticos no Câncer de Boca. R Bras. de Ciên. da Saúde, v. 15, n. 4, p. 471-478, 2011. 


\title{
RECIMA21 - REVISTA CIENTÍFICA MULTIDISCIPLINAR ISSN 2675-6218
}

\begin{abstract}
DETERMINAÇÃO DA INSTABILIDADE CROMOSSÔMICA E MORTE CELULAR EM CÉLULAS DA MUCOSA BUCAL EM PACIENTES COM DEFICIÊNCIA COGNITIVA Isabela Soares Uchôa, Francisco Ariel Paz Santos Freitas, Maria do Amparo Veloso Magalhães, Suzanne Tatianne Teixeira Nascimento, Matheus Fernandes de Castro, Nelson Jorge Carvalho Batista
\end{abstract}

APPOLINÁRIO, Fábio. Dicionário de metodologia científica: um guia para a produção do conhecimento científico. 2. ed. São Paulo: Atlas, 2012.

BRASIL. Resolução no 466, de 12 de dezembro de 2012. Dispõe sobre diretrizes e normas regulamentadoras de pesquisas envolvendo seres humanos. Diário Oficial [da] República Federativa do Brasil, Brasília, DF, 13 jun. 2013.

BRASIL. Resolução no 510, de 07 de abril de 2016. Dispõe sobre as normas aplicáveis a pesquisas em Ciências Humanas e Sociais. Diário Oficial [da] República Federativa do Brasil, Brasília, DF, 24 maio 2016.

CARRANO, A. V.; NATARAJAN, A. T. Considerations for population monitoring using cytogenetic techniques. Mutation Research/Genetic Toxicology, v. 204, n. 3, p. 379-406, 1988.

CHANG, Siow-Wee et al. Feature selection methods for optimizing clinicopathologic input variables in oral cancer prognosis. Asian Pac J Cancer Prev, v. 12, p. 2659-64, 2011.

$\mathrm{CHU}$, Karen P. et al. Head and neck cancer-specific survival based on socioeconomic status in Asians and Pacific Islanders. Cancer, v. 117, n. 9, p. 1935-1945, 2011.

BRASIL. Conselho Nacional de Saúde. Resolução n ${ }^{\circ} 466$, de 12 de dezembro de 2012. Brasília, 2012. Disponível em: http://www.conselho.saude.gov.br/. Acesso em: abr. 2021.

CRUZ, Marília da Nova; HAMDAN, Amer Cavalheiro. O impacto da doença de Alzheimer no cuidador. Psicologia em estudo, v. 13, n. 2, p. 223-229, 2008.

FARIA, Luis Eduardo Meira; BRAGA, Jacqueline Ramos Machado. Aplicação do teste de micronúcleo para avaliação de potencial genotóxico em epitélio oral de estudantes universitários. Rev Eletrôn Atualiza Saúde, v. 1, n. 1, p. 35-41, 2015.

FENECH, Michael et al. HUMN project initiative and review of validation, quality control and prospects for further development of automated micronucleus assays using image cytometry systems. International journal of hygiene and environmental health, v. 216, n. 5, p. 541-552, 2013.

FENECH, Michael; BONASSI, Stefano. The effect of age, gender, diet and lifestyle on DNA damage measured using micronucleus frequency in human peripheral blood lymphocytes. Mutagenesis, v. 26, n. 1, p. 43-49, 2011.

FERREIRA, Aurélio Buarque de Holanda. Dicionário da língua portuguesa. 5. ed. Curitiba: Positivo, 2010.

FREITA, Valéria Souza et al. Efeitos genotóxicos de fatores considerados de risco para o câncer bucal. Revista Baiana de Saúde Pública, v. 29, n. 2, p. 189-189, 2014.

FREITAS, Francisco Ariel Paz Santos; UCHÔA, Isabela Soares; MAGALHÃES, Maria do Amparo Veloso. Importância do Teste de Micronúcleos como Instrumento Preventivo em Saúde Geral e Ambiental. Brazilian Journal of Development, v. 6, n. 9, p. 68530-68542, 2020.

GARBUGLIA, Anna Rosa. Human papillomavirus in head and neck cancer. Cancers, v. 6, n. 3, p. 1705-1726, 2014.

GIL, Antonio Carlos. Como elaborar projetos de pesquisa. 5. ed. São Paulo: Atlas, 2010. 


\title{
RECIMA21 - REVISTA CIENTÍFICA MULTIDISCIPLINAR ISSN 2675-6218
}

\author{
DETERMINAÇÃO DA INSTABILIDADE CROMOSSÔMICA E MORTE CELULAR EM CÉLULAS DA MUCOSA \\ BUCAL EM PACIENTES COM DEFICIÊNCIA COGNITIVA \\ Isabela Soares Uchôa, Francisco Ariel Paz Santos Freitas, Maria do Amparo Veloso Magalhães, \\ Suzanne Tatianne Teixeira Nascimento, Matheus Fernandes de Castro, Nelson Jorge Carvalho Batista
}

GUPTA, Bhawna; JOHNSON, Newell W.; KUMAR, Narinder. Global epidemiology of head and neck cancers: a continuing challenge. Oncology, v. 91, n. 1, p. 13-23, 2016.

GURIAN, Maria Beatriz Ferreira et al. Rastreamento da função cognitiva de idosos nãoinstitucionalizados. Revista Brasileira de Geriatria e gerontologia, v. 15, n. 2, p. 275-284, 2012.

HERRERA, E. M.; LOPEZ, A. D. A.; OSORIO, D. A. P. Cognitive functionality of elderly residents in social protection centers in Cartagena, 2012. Indian J App Res, v. 5, n. 6, p. 19-24, 2015.

INCA - Instituto Nacional do Câncer. Tipos de câncer. Disponível em: http://www2.inca.gov.br/wps/wcm/connect/tiposdecancer/site/home/boca/definicao. Acesso em: abr. 2021.

JOHNSON, Newell W. et al. Global oral health inequalities in incidence and outcomes for oral cancer: causes and solutions. Advances in dental research, v. 23, n. 2, p. 237-246, 2011.

LEITE, Anne Carolina Eleutério; SILVA, Eliete Neves da; MELO, Nilce Santos de. Fatores de risco relacionados com o desenvolvimento do câncer bucal: revisão. Rev. de Clín. Pesq. Odontol., v. 1, n. 3, jan./mar. 2005.

MACÊDO, Francisco Cristiano da Silva; EVANGERLANDY, Gomes Macêdo. Pesquisa: passo a passo para elaboração de trabalhos científicos. Teresina: Macêdo, FCS, 2018.

MANOHARAN, Shanmugam et al. An overview of oral carcinogenesis. International Journal of Nutrition, Pharmacology, Neurological Diseases, v. 6, n. 2, p. 51, 2016.

RETTIG, Eleni M.; SOUZA, Gypsyamber. Epidemiology of head and neck cancer. Surgical Oncology Clinics, v. 24, n. 3, p. 379-396, 2015.

RILEY, Joseph L. et al. Disparities in knowledge of mouth or throat cancer among rural Floridians. The Journal of Rural Health, v. 29, n. 3, p. 294-303, 2013.

SILVA, Valéria Lopes da et al. As dificuldades enfrentadas pelas mães no cuidado à criança com microcefalia. Revista de Divulgação Científica Sena Aires, v. 9, n. 3, p. 394-404, 2020.

SIMARD, Edgar P.; TORRE, Lindsey A.; JEMAL, Ahmedin. International trends in head and neck cancer incidence rates: differences by country, sex and anatomic site. Oral oncology, v. 50, n. 5 , p. 387-403, 2014.

THOMAS, Philip et al. Buccal micronucleus cytome assay. Nature protocols, v. 4, n. 6, p. 825, 2009.

UCHÔA, Isabela Soares; MAGALHÃES, Maria do Amparo Veloso. Teste de micronúcleos como biomarcador para pacientes com patologias diversas: uma revisão integrativa da literatura. Brazilian Journal of Surgery and Clinical Research - BJSCR. v. 27, n. 1, p. 78-83, 2019.

WARNAKULASURIYA, Saman. Living with oral cancer: epidemiology with particular reference to prevalence and life-style changes that influence survival. Oral oncology, v. 46, n. 6, p. 407-410, 2010.

WEATHERSPOON, Darien J. et al. Oral cavity and oropharyngeal cancer incidence trends and disparities in the United States: 2000-2010. Cancer epidemiology, v. 39, n. 4, p. 497-504, 2015. 